\title{
AN INTEGRAL INEQUALITY FOR TWICE DIFFERENTIABLE MAPPINGS AND APPLICATIONS
}

\author{
S. S. DRAGOMIR AND A. SOFO
}

\begin{abstract}
An integral inequality is developed from which when applied to composite quadrature rules in numerical integration it is proved that there is a three fold improvement in the remainder of the classical averages of the Midpoint and Trapezoidal quadratures. Inequalities for special means are also given.
\end{abstract}

\section{Introduction}

In 1976, G. V. Milovanoić and J. E. Pečarić [4] proved a generalization of Ostrowski's inequality for $n$-time differentiable mappings, from which for twice differentiable mappings, we may write:

Theorem 1. Let $f:[a, b] \rightarrow \Re$ be a twice differentiable mapping such that $f^{\prime \prime}$ : $(a, b) \rightarrow \Re$ is bounded on $(a, b)$, i.e., $\|\left. f^{\prime \prime}\right|_{\infty}:=\sup _{t \in(a, b)}\left|f^{\prime \prime}(t)\right|<\infty$. Then we have the inequality

$$
\begin{aligned}
& \left|\frac{1}{2}\left[f(x)+\frac{(x-a) f(a)+(b-x) f(b)}{b-a}\right]-\frac{1}{b-a} \int_{a}^{b} f(t) d t\right| \\
\leq & \frac{(b-a)^{2}}{4}\left[\frac{1}{12}+\frac{\left(x-\frac{a+b}{2}\right)^{2}}{(b-a)^{2}}\right]\left\|f^{\prime \prime}\right\|_{\infty}
\end{aligned}
$$

for all $x \in[a, b]$.

In a more recent paper Cerone, Dragomir and Roumeliotis [1] proved the following inequality:

Theorem 2. Let $f:[a, b] \rightarrow \Re$ be a twice differentiable mapping on $(a, b)$ and $f^{\prime \prime}:(a, b) \rightarrow \Re$ is bounded, i.e., $\left.|| f^{\prime \prime}\right|_{\infty}:=\sup _{t \in(a, b)}\left|f^{\prime \prime}(t)\right|<\infty$. Then we have the inequality

$$
\left|f(x)-\left(x-\frac{a+b}{2}\right) f^{\prime}(x)-\frac{1}{b-a} \int_{a}^{b} f(t) d t\right|
$$

Received July 15, 1999.

2000 Mathematics Subject Classification. Primary 26D15, Secondary 41A55.

Key words and phrases. Integral inequalities, quadrature formulae. 


$$
\leq\left[\frac{(b-a)^{2}}{24}+\frac{1}{2}\left(x-\frac{a+b}{2}\right)^{2}\right]\left\|f^{\prime \prime}\right\|_{\infty} \leq \frac{(b-a)^{2}}{6}\left\|f^{\prime \prime}\right\|_{\infty}
$$

for all $x \in[a, b]$.

Finally, Dragomir and Barnett [2] also prove the following inequality:

Theorem 3. Let $f:[a, b] \rightarrow \Re$ be a twice differentiable mapping on $(a, b)$ and $f^{\prime \prime}:(a, b) \rightarrow \Re$ is bounded, i.e., $\left\|f^{\prime \prime}\right\|_{\infty}:=\sup _{t \in(a, b)}\left|f^{\prime \prime}(t)\right|<\infty$. Then we have the inequality

$$
\begin{aligned}
& \left|f(x)-\frac{f(b)-f(a)}{b-a}\left(x-\frac{a+b}{2}\right)-\frac{1}{b-a} \int_{a}^{b} f(t) d t\right| \\
\leq & \frac{(b-a)^{2}}{2}\left\{\left[\left(\frac{x-\frac{a+b}{2}}{b-a}\right)^{2}+\frac{1}{4}\right]^{2}+\frac{1}{12}\right\}\left\|f^{\prime \prime}\right\|_{\infty} \leq \frac{(b-a)^{2}}{6}\left\|f^{\prime \prime}\right\|_{\infty}
\end{aligned}
$$

for all $x \in[a, b]$.

In this paper we point out an integral inequality, similar in a sense, to that of Cerone, Dragomir and Roumeliotis [1], or Dragomir and Barnett [2], and apply it to special means and numerical integration. We begin with the following result.

\section{An Integral Inequality}

The following theorem is now proved and subsequently applied to numerical integration and special means.

Theorem 4. Let $g:[a, b] \rightarrow \Re$ be a mapping whose first derivative is absolutely continuous on $[a, b]$ and assume that the second derivative $g^{\prime \prime} \in L_{\infty}[a, b]$. Then we have the inequality

$$
\begin{aligned}
& \left|\int_{a}^{b} g(t) d t-\frac{1}{2}\left[g(x)+\frac{g(a)+g(b)}{2}\right](b-a)+\frac{(b-a)}{2}\left(x-\frac{a+b}{2}\right) g^{\prime}(x)\right| \\
\leq & \left\|g^{\prime \prime}\right\|_{\infty}\left(\frac{1}{3}\left|x-\frac{a+b}{2}\right|^{3}+\frac{(b-a)^{3}}{48}\right)
\end{aligned}
$$

for all $x \in[a, b]$.

Proof. Let us start with the following integral equality

$$
f(x)=\frac{1}{b-a}\left(\int_{a}^{b} f(t) d t+\int_{a}^{b} p(x, t) f^{\prime}(t) d t\right)
$$


for all $x \in[a, b]$, provided $f$ is absolutely continuous on $[a, b]$, and the kernel $p:[a, b]^{2} \rightarrow \Re$ is given by

$$
p(x, t):= \begin{cases}t-a & \text { if } t \in[a, x] \\ t-b & \text { if } t \in(x, b]\end{cases}
$$

where $t \in[a, b]$. A simple proof using the integration by parts formula can be found in Dragomir and Wang [3]. Now choose in $(2.2), f(x)=\left(x-\frac{a+b}{2}\right) g^{\prime}(x)$ to get

$$
\begin{aligned}
& \left(x-\frac{a+b}{2}\right) g^{\prime}(x) \\
= & \frac{1}{b-a}\left(\int_{a}^{b}\left(t-\frac{a+b}{2}\right) g^{\prime}(t) d t+\int_{a}^{b} p(x, t)\left[g^{\prime}(t)+\left(t-\frac{a+b}{2}\right) g^{\prime \prime}(t)\right] d t\right) .
\end{aligned}
$$

Integrating by parts we have

$$
\int_{a}^{b}\left(t-\frac{a+b}{2}\right) g^{\prime}(t) d t=\frac{(g(a)+g(b))(b-a)}{2}-\int_{a}^{b} g(t) d t .
$$

Also upon using (2.2), we have that

$$
\begin{aligned}
& \int_{a}^{b} p(x, t)\left[g^{\prime}(t)+\left(t-\frac{a+b}{2}\right) g^{\prime \prime}(t)\right] d t \\
= & \int_{a}^{b} p(x, t) g^{\prime}(t) d t+\int_{a}^{b} p(x, t)\left(t-\frac{a+b}{2}\right) g^{\prime \prime}(t) d t \\
= & (b-a) g(x)-\int_{a}^{b} g(t) d t+\int_{a}^{b} p(x, t)\left(t-\frac{a+b}{2}\right) g^{\prime \prime}(t) d t .
\end{aligned}
$$

Now by (2.3), (2.4) and (2.5) we deduce that

$$
\begin{aligned}
(b-a)\left(x-\frac{a+b}{2}\right) g^{\prime}(x)= & \frac{(g(a)+g(b))(b-a)}{2}-\int_{a}^{b} g(t) d t+(b-a) g(x)-\int_{a}^{b} g(t) d t \\
& +\int_{a}^{b} p(x, t)\left(t-\frac{a+b}{2}\right) g^{\prime \prime}(t) d t
\end{aligned}
$$

from where we get the identity

$$
\begin{aligned}
\int_{a}^{b} g(t) d t= & \frac{1}{2}\left[g(x)+\frac{g(a)+g(b)}{2}\right](b-a) \\
& -\frac{(b-a)}{2}\left(x-\frac{a+b}{2}\right) g^{\prime}(x)+\frac{1}{2} \int_{a}^{b} p(x, t)\left(t-\frac{a+b}{2}\right) g^{\prime \prime}(t) d t
\end{aligned}
$$

for all $x \in[a, b]$. Now using $(2.6)$ we have

$$
\begin{aligned}
& \left|\int_{a}^{b} g(t) d t-\frac{1}{2}\left[g(x)+\frac{g(a)+g(b)}{2}\right](b-a)+\frac{(b-a)}{2}\left(x-\frac{a+b}{2}\right) g^{\prime}(x)\right| \\
\leq & \frac{1}{2} \int_{a}^{b}|p(x, t)|\left|t-\frac{a+b}{2}\right| g^{\prime \prime}(t) \mid d t .
\end{aligned}
$$


Obviously, we have

$$
\int_{a}^{b}|p(x, t)|\left|t-\frac{a+b}{2}\right|\left|g^{\prime \prime}(t)\right| d t \leq\left\|g^{\prime \prime}\right\|_{\infty} \int_{a}^{b}|p(x, t)|\left|t-\frac{a+b}{2}\right| d t
$$

where $\left\|g^{\prime \prime}\right\|_{\infty}:=\sup _{t \in(a, b)}\left|g^{\prime \prime}(t)\right|<\infty$. Also

$$
I:=\int_{a}^{b}|p(x, t)|\left|t-\frac{a+b}{2}\right| d t=\int_{a}^{x}(t-a)\left|t-\frac{a+b}{2}\right| d t+\int_{x}^{b}(b-t)\left|t-\frac{a+b}{2}\right| d t .
$$

We have two cases:

a). For $x \in\left[a, \frac{a+b}{2}\right]$ we obtain

$$
I=\int_{a}^{x}(t-a)\left(\frac{a+b}{2}-t\right) d t+\int_{x}^{\frac{a+b}{2}}(b-t)\left(\frac{a+b}{2}-t\right) d t+\int_{\frac{a+b}{2}}^{b}(b-t)\left(t-\frac{a+b}{2}\right) d t
$$

We have

and

$$
\begin{aligned}
& I_{1}=\int_{a}^{x}(t-a)\left(\frac{a+b}{2}-t\right) d t=\frac{(a+3 b-4 x)(x-a)^{2}}{12} \\
& I_{2}=\int_{x}^{\frac{a+b}{2}}(b-t)\left(\frac{a+b}{2}-t\right) d t=\frac{(5 b-a-4 x)(2 x-a-b)^{2}}{48}
\end{aligned}
$$

$$
I_{3}=\int_{\frac{a+b}{2}}^{b}(b-t)\left(t-\frac{a+b}{2}\right) d t=\frac{(b-a)^{3}}{48}
$$

and hence, upon simplification

$$
I=\frac{2}{3}\left(\frac{a+b}{2}-x\right)^{3}+\frac{(b-a)^{3}}{24}, \quad x \in\left[a, \frac{a+b}{2}\right]
$$

b). For $x \in\left(\frac{a+b}{2}, b\right]$ we obtain

$$
\begin{aligned}
I= & \int_{a}^{\frac{a+b}{2}}(t-a)\left(\frac{a+b}{2}-t\right) d t+\int_{\frac{a+b}{2}}^{x}(t-a)\left(t-\frac{a+b}{2}\right) d t \\
& +\int_{x}^{b}(b-t)\left(t-\frac{a+b}{2}\right) d t \\
= & \frac{(b-a)^{3}}{48}+\frac{(4 x-5 a+b)(2 x-a-b)^{2}}{48}+\frac{(4 x-3 a-b)(x-b)^{2}}{12} \\
= & \frac{2}{3}\left(x-\frac{a+b}{2}\right)^{3}+\frac{(b-a)^{3}}{24},
\end{aligned}
$$

and referring to $(2.7)$, we obtain the result (2.1) of Theorem 4. 
Remark 1. In (2.1) if we investigate the end points $x=a, x=b$ and the midpoint $x=\frac{a+b}{2}$ we find that the midpoint gives us the best estimator, so that we have

$$
\left|\frac{1}{b-a} \int_{a}^{b} g(t) d t-\frac{1}{2}\left[g\left(\frac{a+b}{2}\right)+\frac{g(a)+g(b)}{2}\right]\right| \leq\left\|g^{\prime \prime}\right\|_{\infty} \frac{(b-a)^{2}}{48} .
$$

\section{Application to Composite Quadrature Rules}

We may utilize the previous inequality to give us estimates of composite quadrature rules which, it turns out have a markedly smaller error than that which may be obtained by the classical results.

Theorem 5. Let $I_{n}: a=x_{0}<x_{1}<\cdots<x_{n-1}<x_{n}=b$ be a partition of the interval $[a, b], h_{i}=x_{i+1}-x_{i}, \nu(h):=\max \left\{h_{i}: i=1, \ldots, n\right\}, \xi_{i} \in\left[x_{i}, x_{i+1}\right]$,

$$
S\left(g, I_{n}, \xi\right)=\frac{1}{2} \sum_{i=0}^{n-1}\left[g\left(\xi_{i}\right)+\frac{g\left(x_{i}\right)+g\left(x_{i+1}\right)}{2}\right] h_{i}-\frac{1}{2} \sum_{i=0}^{n-1}\left(\xi_{i}-\frac{x_{i}+x_{i+1}}{2}\right) h_{i} g^{\prime}\left(\xi_{i}\right)
$$

then

$$
\int_{a}^{b} g(x) d x=S\left(g, I_{n}, \xi\right)+R\left(g, I_{n}, \xi\right)
$$

and

$$
\left|R\left(g, I_{n}, \xi\right)\right| \leq\left\|g^{\prime \prime}\right\|_{\infty}\left[\frac{1}{3} \sum_{i=0}^{n-1}\left|\xi_{i}-\frac{x_{i}+x_{i+1}}{2}\right|^{3}+\frac{1}{48} \sum_{i=0}^{n-1} h_{i}^{3}\right]
$$

Proof. Applying (2.1) on $\xi_{i} \in\left[x_{i}, x_{i+1}\right]$ we have

$$
\begin{aligned}
& \left|\int_{x_{i}}^{x_{i+1}} g(t) d t-\frac{1}{2}\left[g\left(\xi_{i}\right)+\frac{g\left(x_{i}\right)+g\left(x_{i+1}\right)}{2}\right] h_{i}+\frac{1}{2}\left(\xi_{i}-\frac{x_{i}+x_{i+1}}{2}\right) h_{i} g^{\prime}\left(\xi_{i}\right)\right| \\
\leq & \left\|g^{\prime \prime}\right\|_{\infty}\left(\frac{1}{3}\left|\xi_{i}-\frac{x_{i}+x_{i+1}}{2}\right|^{3}+\frac{h_{i}^{3}}{48}\right) .
\end{aligned}
$$

Now summing over $i$ from 0 to $n-1$ and utilizing the triangle inequality, we have

$$
\begin{aligned}
& \left|\int_{a}^{b} g(t) d t-S\left(g, I_{n}, \xi\right)\right| \\
= & \left|\sum_{i=0}^{n-1} \int_{x_{i}}^{x_{i+1}} g(t) d t\right|-\sum_{i=0}^{n-1} h_{i}\left|\frac{1}{2}\left[g\left(\xi_{i}\right)+\frac{g\left(x_{i}\right)+g\left(x_{i+1}\right)}{2}\right]+\frac{1}{2}\left(\xi_{i}-\frac{x_{i}+x_{i+1}}{2}\right) g^{\prime}\left(\xi_{i}\right)\right| \\
\leq & \left\|g^{\prime \prime}\right\|_{\infty} \sum_{i=0}^{n-1}\left(\frac{1}{3}\left|\xi_{i}-\frac{x_{i}+x_{i+1}}{2}\right|^{3}+\frac{h_{i}^{3}}{48}\right)
\end{aligned}
$$


and therefore

$$
\left|R\left(g, I_{h}, \xi\right)\right| \leq\left\|g^{\prime \prime}\right\|_{\infty}\left(\frac{1}{3} \sum_{i=0}^{n-1}\left|\xi_{i}-\frac{x_{i}+x_{i+1}}{2}\right|^{3}+\frac{1}{48} \sum_{i=0}^{n-1} h_{i}^{3}\right)
$$

Corollary 1. If $\xi_{i}=\frac{x_{i}+x_{i+1}}{2}$ then

$$
\bar{S}\left(g, I_{n}\right)=\frac{1}{2} \sum_{i=0}^{n-1}\left[g\left(\frac{x_{i}+x_{i+1}}{2}\right)+\frac{g\left(x_{i}\right)+g\left(x_{i+1}\right)}{2}\right] h_{i}
$$

and

$$
\left|\bar{R}\left(g, I_{n}\right)\right| \leq \frac{\left\|g^{\prime \prime}\right\|_{\infty}}{48} \sum_{i=0}^{n-1} h_{i}^{3} .
$$

$\bar{S}\left(g, I_{n}\right)$ may be thought of as the arithmetic mean of the Midpoint and the Trapezoidal quadrature rules.

Remark 2. However it is clear that inequality (3.1) is much better than the classical averages of the remainders of the Midpoint and Trapezoidal quadratures. Consider

$$
\int_{a}^{b} f(x) d x=A_{T}\left(f, I_{h}\right)+R_{T}\left(f, I_{h}\right)
$$

where $A_{T}\left(f, I_{h}\right)$ is the Trapezoidal rule

$$
A_{T}\left(f, I_{h}\right):=\sum_{i=0}^{n-1}\left[\frac{f\left(x_{i}\right)+f\left(x_{i+1}\right)}{2}\right] h_{i}
$$

and the remainder term $R_{T}\left(f, I_{h}\right)$ satisfies

$$
\left|R_{T}\left(f, I_{h}\right)\right| \leq \frac{\left\|f^{\prime \prime}\right\|_{\infty}}{12} \sum_{i=0}^{n-1} h_{i}^{3} .
$$

Also

$$
\int_{a}^{b} f(x) d x=A_{M}\left(f, I_{h}\right)+R_{M}\left(f, I_{h}\right)
$$

where

$$
A_{M}\left(f, I_{h}\right):=\sum_{i=0}^{n-1}\left[f\left(\frac{x_{i}+x_{i+1}}{2}\right)\right] h_{i}
$$

is the Midpoint quadrature rule and the remainder term $R_{M}\left(f, I_{h}\right)$ satisfies the estimation

$$
\left|R_{M}\left(f, I_{h}\right)\right| \leq \frac{\left\|f^{\prime \prime}\right\|_{\infty}}{24} \sum_{i=0}^{n-1} h_{i}^{3}
$$


Now we see from (3.2) (3.3) (3.4) and (3.5)

$$
\int_{a}^{b} f(x) d x=\frac{1}{2}\left(A_{M}\left(f, I_{h}\right)+A_{T}\left(f, I_{h}\right)+R_{M}\left(f, I_{h}\right)+R_{T}\left(f, I_{h}\right)\right),
$$

from the remainders and using the triangle inequality we have

$$
\begin{aligned}
\frac{1}{2}\left|R_{M}\left(f, I_{h}\right)+R_{T}\left(f, I_{h}\right)\right| & \leq \frac{1}{2}\left|R_{M}\left(f, I_{h}\right)\right|+\frac{1}{2}\left|R_{T}\left(f, I_{h}\right)\right| \\
& \leq \frac{\left\|f^{\prime \prime}\right\|_{\infty}}{24} \sum_{i=0}^{n-1} h_{i}^{3}+\frac{\left\|f^{\prime \prime}\right\|_{\infty}}{48} \sum_{i=0}^{n-1} h_{i}^{3} \\
& =\frac{\left\|f^{\prime \prime}\right\|_{\infty}}{16} \sum_{i=0}^{n-1} h_{i}^{3} .
\end{aligned}
$$

It can be clearly seen that (3.1) is a three fold better estimator than (3.6).

The conclusion of Corollary 1 can also be obtained from the Milovanović-Pečarić result, Theorem 1, upon setting $x=\frac{a+b}{2}$.

\section{Applications for Some Special Means}

Let us recall the following means:

(a) The Arithmetic mean:

$$
A=A(a, b):=\frac{a+b}{2}, \quad a, b \geq 0 .
$$

(b) The Geometric mean:

$$
G=G(a, b):=\sqrt{a b}, \quad a, b \geq 0 .
$$

(c) The Harmonic mean:

$$
H=H(a, b):=\frac{2}{\frac{1}{a}+\frac{1}{b}}, \quad a, b>0 .
$$

(d) The Logarithmic mean:

$$
L=L(a, b):=\left\{\begin{array}{ll}
a & \text { if } a=b \\
\frac{b-a}{\ln b-\ln a} & \text { if } a \neq b
\end{array}, \quad a, b>0 .\right.
$$

(e) The Identric mean:

$$
I=I(a, b):=\left\{\begin{array}{ll}
a & \text { if } a=b \\
\frac{1}{e}\left(\frac{b^{b}}{a^{a}}\right)^{\frac{1}{b-a}} & \text { if } a \neq b
\end{array}, \quad a, b>0 .\right.
$$


(f) The p-logarithmic mean:

$$
L_{p}=L_{p}(a, b):=\left\{\begin{array}{ll}
a & \text { if } a=b \\
{\left[\frac{b^{p+1}-a^{p+1}}{(p+1)(b-a)}\right]^{\frac{1}{p}}} & \text { if } a \neq b
\end{array}, \quad a, b>0 .\right.
$$

where $p \in \Re \backslash\{-1,0\}$. The following is well known in the literature:

$$
H \leq G \leq L \leq I \leq A
$$

It is also well known that $L_{p}$ is monotonically increasing over $p \in \Re$ (assuming that $L_{0}:=I$ and $\left.L_{-1}:=L\right)$.

The inequality (2.1) may be rewritten as

$$
\begin{aligned}
& \left|-(x-A(a, b)) g^{\prime}(x)+\frac{1}{2}\left[g(x)+\frac{g(a)+g(b)}{2}\right]-\frac{1}{b-a} \int_{a}^{b} g(t) d t\right| \\
\leq & \left\|g^{\prime \prime}\right\|_{\infty}\left(\frac{1}{3(b-a)}|x-A(a, b)|^{3}+\frac{(b-a)^{2}}{48}\right) .
\end{aligned}
$$

We may now apply (4.1) to deduce some inequalities for special means given above, by the use of some particular mappings as follows.

(1) Consider $g(x)=\ln x, x \in[a, b] \subset(0, \infty)$ then

$$
\begin{aligned}
\frac{1}{b-a} \int_{a}^{b} g(t) d t & =\ln I(a, b), \\
\frac{g(a)+g(b)}{2} & =\ln G(a, b)
\end{aligned}
$$

and

$$
\left\|g^{\prime \prime}\right\|_{\infty}=\sup _{t \in(a, b)}\left|g^{\prime \prime}(t)\right|=\frac{1}{a^{2}}
$$

From (4.1) we have that

$$
\left|\frac{A(a, b)}{x}-1+\ln x+\ln G(a, b)-2 \ln I(a, b)\right| \leq \frac{2}{a^{2}}\left(\frac{1}{3(b-a)}|x-A(a, b)|^{3}+\frac{(b-a)^{2}}{48}\right)
$$

form which we obtain the best estimate at the centre point $x=\frac{a+b}{2}$, so that

$$
|\ln A(a, b)+\ln G(a, b)-2 \ln I(a, b)| \leq \frac{(b-a)^{2}}{24 a^{2}}
$$

or

$$
\left|\ln \left(\frac{A G}{I^{2}}\right)\right| \leq \frac{(b-a)^{2}}{24 a^{2}}
$$


(2) Consider $g(x)=\frac{1}{x}, x \in[a, b] \subset(0, \infty)$ then

$$
\begin{aligned}
\frac{1}{b-a} \int_{a}^{b} g(t) d t & =L^{-1}(a, b), \\
\frac{g(a)+g(b)}{2} & =\frac{A(a, b)}{G^{2}(a, b)}
\end{aligned}
$$

and

$$
\left\|g^{\prime \prime}\right\|_{\infty}=\sup _{t \in(a, b)}\left|g^{\prime \prime}(t)\right|=\frac{2}{a^{3}}
$$

From (4.1) we have that

$$
\left|\frac{1}{x}\left(3-\frac{A(a, b)}{x}\right)+\frac{A(a, b)}{G^{2}(a, b)}-L^{-1}(a, b)\right| \leq \frac{2}{a^{3}}\left(\frac{1}{3(b-a)}|x-A(a, b)|^{3}+\frac{(b-a)^{2}}{48}\right)
$$

and the best estimate is obtained at the centre point $x=\frac{a+b}{2}$, so that

$$
\left|\frac{1}{A(a, b)}+\frac{A(a, b)}{G^{2}(a, b)}-L^{-1}(a, b)\right| \leq \frac{(b-a)^{2}}{24 a^{3}} .
$$

(3) Consider $g(x)=x^{p} g:(0, \infty) \rightarrow \Re$ where $p \in \Re \backslash\{-1,0\}$ then for $a<b$

$$
\begin{aligned}
\frac{1}{b-a} \int_{a}^{b} g(t) d t & =L_{p}^{p}(a, b), \\
\frac{g(a)+g(b)}{2} & =A\left(a^{p}, b^{p}\right)
\end{aligned}
$$

and

$$
\left\|g^{\prime \prime}\right\|_{\infty}=|p(p-1)| \cdot\left\{\begin{array}{ll}
b^{p-2} & \text { if } p \in[2, \infty) \\
a^{p-2} & \text { if } p \in(-\infty, 2) \backslash\{1,0\}
\end{array} .\right.
$$

From (4.1) we have that

$$
\begin{aligned}
& \left|\frac{x^{p-1}}{2}((1-p) x+2 p A(a, b))+\frac{A\left(a^{p}, b^{p}\right)}{2}-L_{p}^{p}(a, b)\right| \\
\leq & |p(p-1)| \delta_{p}(a, b)\left(\frac{1}{3(b-a)}|x-A(a, b)|^{3}+\frac{(b-a)^{2}}{48}\right)
\end{aligned}
$$

where

$$
\delta_{p}(a, b):=\left\{\begin{array}{ll}
b^{p-2} & \text { if } p \in[2, \infty) \\
a^{p-2} & \text { if } p \in(-\infty, 2) \backslash\{-1,0\}
\end{array} .\right.
$$

At $x=\frac{a+b}{2}$ the best estimate is

$$
\left|A^{p}(a, b)+A\left(a^{p}, b^{p}\right)-2 L_{p}^{p}(a, b)\right| \leq|p(p-1)| \delta_{p}(a, b) \frac{(b-a)^{2}}{24} .
$$




\section{References}

[1] P. Cerone, S. S. Dragomir and J. Roumeliotis, An inequality of ostrowski type for mappings whose second derivatives are bounded and applications, RGMIA Research Report Collection, V.U.T., 1(1998), 33-39.

[2] S. S. Dragomir and N. S. Barnett, An ostrowski type inequality for mappings whose second derivatives are bounded and applications, RGMIA Research Report Collection, V.U.T., 1(1999), 67-76.

[3] S. S. Dragomir and S. Wang, Application of Ostrowshi's inequality to the estimate of error bounds for some special means and some numerical quadrature rules, Appl. Math. Lett., 11(1998), 105-109.

[4] D. S. Mitrinović, J. E. Pečarić and A. M. Fink, Inequalities for Functions and Their Integrals and Derivatives, Kluwer Academic, Dordrecht, 1994.

School of Communications and Informatics, Victoria University of Technology, Melbourne, Australia.

E-mail: sever@matilda.vu.edu.au

School of Communications and Informatics, Victoria University of Technology, Melbourne, Australia.

E-mail: sofo@matilda.vu.edu.au 